\title{
Estudo da presença de síndrome metabólica e relação com o histórico familiar em escolares
}

\section{Study of the presence of metabolic syndrome and its relationship with the familiar history in students}

\author{
Sandra Maria Barbalho ${ }^{1 *}$; Adriana Mitie Kawakubo ${ }^{2}$; Karen Gisele Ferreira de \\ Souza $^{3}$; João Gilberto Traldi Junior ${ }^{4}$; Claudemir Gregório Mendes ${ }^{5}$; Fábio Maurizio \\ Nery $^{6}$, Márcia Gabaldi Rocha ${ }^{7}$; Ricardo de Alvares Goulart ${ }^{8}$
}

\section{Resumo}

Este trabalho tem por objetivo avaliar a prevalência de Síndrome Metabólica (SM) e a influência do histórico familiar em estudantes de escolas públicas e privadas. Foram avaliados 337 alunos de 2 escolas públicas e 2 particulares do Município de Marília-SP. Foram colhidos dados de peso, altura, índice de massa corpórea, circunferência da cintura, pressão arterial e aferições bioquímicas em jejum de glicemia, colesterol total, HDL-c, LDL-c e triglicerídeos. Observou-se que dos 337 alunos avaliados, 18,4\% apresentam a SM. Dos portadores de SM: 92,8\% nas escolas particulares apresentavam histórico familiar e $85,3 \%$ nas públicas. Do total de alunos estudados, $15,4 \%$ eram obesos e 54,0\% sedentários. A alta prevalência da SM nos adolescentes indica a possibilidade de apresentarem complicações cardíacas na fase adulta. Sendo assim, são necessárias medidas intervencionistas urgentes, abordadas por equipe multidisciplinar para que este grupo etário seja assistido integralmente para que se tornem indivíduos adultos mais saudáveis.

Palavras-Chave: Adolescente. Obesidade. Glicemia. Lipídeos.

\begin{abstract}
This work had as aim to evaluate the predominance of Metabolic Syndrome (SM) and the influence of the familiar historical at adolescents of public and private schools. There were studied 337 pupils of public and private schools in the city of Marília - SP. The following data were evaluated: IMC, waist circumference, blood pressure and biochemical profile (glycemia, total cholesterol, HDL-c, LDL-c and triglycerides and $18,4 \%$ of the pupils already presented the SM. Of the bearers of SM in the private schools, $92,8 \%$ presented familiar historical and $85,3 \%$ in the public ones; $15,4 \%$ of the adolescent were classified as obese and a 54,0\% as sedentary people. The presence of SM in the adolescents indicates the possibility to present cardiac complications in the adult phase. It is necessary that government starts an interventionist work boarded by multidiscipline team to assist this group so that they become healthier adult individuals.
\end{abstract}

Keywords: Adolescent. Obesity. Blood glucose. Lipids.

\footnotetext{
${ }^{1}$ PhD, Professora da Faculdade de Medicina de Marília (UNIMAR) e da Faculdade de Tecnologia de Alimentos de Marília (FATEC). Alameda dos Jatobás, 126 Bairro Santa Gertrudes, Marília, SP, CEP 17.514-844. Tel. (14) 3306-9434/(14) 9655-3190.

*Autor para correspondência: smbarbalho@terra.com.br

${ }^{2}$ Médico, Faculdade de Medicina de Marília, UNIMAR, Marília, SP. E-mail: akawakubo@yahoo.com.br

${ }^{3}$ Médico, Faculdade de Medicina de Marília, FAMEMA, Marília, SP. E-mail: karengisele@uol.com.br

${ }^{4}$ Médico, UNIMAR. E-mail: gtraldij@hotmail.com

${ }^{5}$ Médico, UNIMAR. E-mail: fabionery@gmail.com

${ }^{6}$ Biomédico, Professor da UNIMAR. E-mail: cgremen@yahoo.com.br

${ }^{7}$ Mestre, Professora da UNIMAR. E-mail: marciagabaldi@hotmail.com

${ }^{8}$ Médico, UNIMAR. E-mail: ricardogoulartmed@hotmail.com
} 


\section{Introdução}

A Síndrome Metabólica (SM) representa a anormalidade metabólica mais comum da atualidade e também é a maior responsável por eventos cardiovasculares na população (BORNEO; LEÓN, 2012). Seu desenvolvimento em um indivíduo depende da interação complexa entre a predisposição genética e fatores ligados ao estilo de vida, como padrão dietético, sedentarismo e obesidade $(\mathrm{OH}$ et al., 2012).

Uma das definições de SM mais frequentemente utilizada é proposta pelo Expert Panel on Detection, Evaluation, and Treatment of High Blood Cholesterol in Adults (National Cholesterol Education Program Adult Treatment Panel III) (2001) e está baseada na presença de três ou mais fatores de risco cardiovascular em um indivíduo: obesidade visceral, intolerância à glicose, dislipidemia (elevação dos níveis de triglicerídeos no plasma e diminuição dos níveis de HDL-c) e aumento da pressão arterial sistêmica (HAS) (DE MARCO et al., 2012).

As transformações ocorridas nos padrões socioeconômicos e culturais da população a partir da segunda metade do século XX contribuíram para aumentar e melhorar a vida do homem, mas por outro lado, incrementaram mudanças nos hábitos alimentares e no gasto energético relacionado às atividades diárias e físicas que influenciam de forma importante o processo saúde-doença. Associado a isto está o estresse inerente ao estilo de vida moderno que pode contribuir com a obesidade, o Diabetes Mellitus e a HAS, que frequentemente desencadeiam as dislipidemias e aumentam o risco para as doenças cardiovasculares (DCV) (EL MESALLAMY et al., 2010).

A obesidade é considerada um problema de saúde pública que leva a sérias consequências sociais, psicológicas e físicas e está associada ao maior risco de morbimortalidade por enfermidades crônicas não-transmissíveis. Em estudos epidemiológicos, o ganho ponderal é um fator de risco independente para o desenvolvimento da síndrome metabólica e, portanto, para doenças vasculares (SÁNCHEZGÓMEZ et al., 2012).

O Brasil e outros muitos países do mundo apresentam um quadro preocupante em relação às $\mathrm{DCV}$, não só pelas elevadas taxas de morbimortalidade, mas principalmente por elas estarem afetando de forma importante os estratos etários mais jovens (STEVENS; KING; SHIBUYA, 2010). É essencial que sejam delineados métodos práticos de identificação desta síndrome que é inicialmente silenciosa nas crianças e nos jovens.

Dentro deste contexto, este estudo teve por objetivo avaliar a prevalência de Síndrome SM e a influência do histórico familiar em estudantes de escolas públicas e privadas.

\section{Material e método}

Este estudo foi realizado após obtenção do parecer favorável do Comitê de Ética em Pesquisa da Universidade de Marília UNIMAR (protocolo 2500000764/2007-47) e após os responsáveis pelos alunos assinarem o termo de consentimento (Resolução 196/10 de Outubro de 1996 - Conselho Nacional de Saúde - CNS).

A cidade de Marília SP, segundo a Diretoria de Ensino da Região de Marília, possui 41 escolas públicas e 20 particulares. O local de estudo envolveu duas escolas públicas e duas privadas desta cidade, sendo duas da região central da cidade e outras duas da periferia. Várias escolas foram procuradas para a execução do trabalho, mas apenas as 4 estudadas manifestaram interesse no estudo.

Dos dois colégios particulares a amostragem foi de 206 alunos, 123 do sexo feminino e 83 do sexo masculino. Já das escolas públicas o número estudado foi de 131, sendo 75 do sexo feminino e 56 do sexo masculino. Estes alunos foram escolhidos aleatoriamente e a faixa etária envolvida foi entre 14 e 18 anos. A amostragem inicial dos estudantes 
era de 250 em escolas públicas e 250 nas privadas, mas muitos desistiram de participar no dia da coleta de sangue.

As medidas antropométricas foram tomadas e avaliadas conforme a metodologia proposta por Heyward (2000). O peso e a altura, para cálculo do Índice de massa Corpórea (IMC), foram aferidos respectivamente, por meio do uso de balança eletrônica com capacidade de $180 \mathrm{~kg}$ e de um estadiômetro com precisão em milímetros. A Circunferência da Cintura (CC) (medida no espaço médio abdominal entre a crista ilíaca e o rebordo costal inferior) foi realizada segundo a I Diretriz de Prevenção da Aterosclerose na Infância e na Adolescência (SOCIEDADE BRASILEIRA DE CARDIOLOGIA, 2005).

A Pressão Arterial (PA) foi aferida nos dois membros superiores no paciente em repouso, sentado com o braço na altura do coração com a diferença de 5 minutos de aferição de um braço para outro. Após as aferições foram analisadas as pressões arteriais segundo o percentil (Altura/Peso) de acordo com parâmetros do National High Blood Pressure Education Program Working Group on high Blood pressure in children and Adolescents (SOCIEDADE BRASILEIRA DE CARDIOLOGIA, 2007).

Foram realizados, após jejum de 12 horas, exames de glicemia, colesterol total, HDL-c, LDL-c e triglicerídeos no Laboratório de Análises Clínicas do Hospital Universitário da Universidade de Marília (Laboratório São Francisco). Os resultados foram interpretados segundo a I Diretriz de Prevenção da Aterosclerose na Infância e na Adolescência da Sociedade Brasileira de Cardiologia (2005).

Para ser classificado como portador de SM, o estudante deve ter apresentado pelo menos 3 fatores de risco, como por exemplo obesidade, hiperglicemia e baixos níveis de HDL-c (BORNEO;
LEÓN, 2012).

Os dados acerca do histórico familiar de desenvolver DCV foram obtidos a partir da análise das respostas de um questionário com questões sobre presença de diabetes, hipertensão, dislipidemias e doenças cardíacas em pais e avós maternos e paternos. O questionário foi respondido pelos próprios alunos (no dia anterior à coleta de dados foi solicitado que consultassem seus pais para responderem ao questionário de forma mais fidedigna).

Baseando-se no questionário, considerou-se como sendo sedentário o adolescente que relatou não praticar atividade física nenhuma vez na semana.

Os dados obtidos foram analisados por Estatística Descritiva e testes paramétricos comparando os dois momentos do estudo.

\section{Resultados}

A tabela 1 mostra os resultados dos parâmetros de IMC, CC, PA e perfil glicêmico e lipídico. Pode-se observar na tabela 1 que houve diferença significativa na PA diastólica esquerda e direita quando se compara o gênero, sendo que os valores maiores são do sexo feminino. Também houve diferença significativa nos valores de glicemia, podendo-se observar que indivíduos do sexo feminino apresentaram médias mais elevadas quando comparados aos do sexo masculino.

Quanto aos níveis de HDL-c, pôde-se observar que os indivíduos do sexo masculino mostraram níveis mais elevados quando são comparados ao sexo feminino. 
Tabela1 - Resultados dos valores de IMC (índice de massa corpórea), CC (circunferência da cintura), PA (pressão arterial), glicemia (Gli), colesterol total (Col) e frações (HDL-c e LDL-c) e triglicerídeos (TGC)

\begin{tabular}{lccccccccccc}
\hline Sexo & IMC & CC & PAsd* $^{*}$ & PAse & PAdd & PAde $\ddagger$ & Gli & Col & LDLc & HDLc & TGC \\
\hline \multirow{2}{*}{26,2} & 90,0 & 118,3 & 72,9 & 118,6 & 71,9 & 85,9 & 171,1 & 101,8 & 46,4 & 114,3 \\
& $\pm 4,1$ & $\pm 11,5$ & $\pm 11,3$ & $\pm 10,9$ & $\pm 10,5$ & $\pm 10,6$ & $\pm 8,7$ & $\pm 32,2$ & $\pm 26,5$ & $\pm 11,3$ & $\pm 47,7$ \\
& & & & & & & & & & & \\
\multirow{2}{*}{} & 25,1 & 88,7 & 121,5 & 67,3 & 120,7 & 68,5 & 82,5 & 178,6 & 102,0 & 50,5 & 130,2 \\
& $\pm 4,7$ & $\pm 11,9$ & $\pm 11,4$ & $\pm 8,3$ & $\pm 11,5$ & $\pm 8,0$ & $\pm 7,2$ & $\pm 28,0$ & $\pm 22,7$ & $\pm 8,7$ & $\pm 55,6$
\end{tabular}

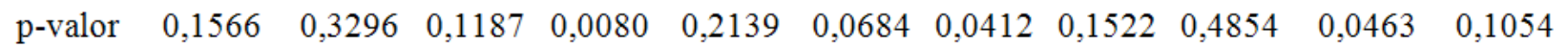

*PAsd: pressão arterial sistólica no braço direito; \#PAse: pressão arterial sistólica no braço esquerdo; †PAdd: pressão arterial diastólica no braço direito; †PAde: pressão arterial diastólica no braço esquerdo.

Fonte: Autores

A tabela 2 mostra a porcentagem de alunos das escolas estudadas que apresentaram Síndrome metabólica (SM) de acordo a I Diretriz de Prevenção da Aterosclerose na Infância e na Adolescência (SOCIEDADE BRASILEIRA DE CARDIOLOGIA, 2005). O número de indivíduos acometidos de SM nas escolas públicas $(53,9 \%)$ variou significativamente quando se fez a comparação com as escolas privadas $(28,3 \%)$, sendo que nestas últimas encontraram-se as menores médias ( $\mathrm{p}$-valor $=$ $0,0043)$.

Tabela 2 - Porcentagem de alunos (de ambos os sexos) das escolas privadas e públicas segundo a presença de Síndrome Metabólica (SM).

\begin{tabular}{|c|c|c|c|c|}
\hline \multirow{2}{*}{ Escola } & \multirow{2}{*}{ Sexo } & \multicolumn{2}{|c|}{ Presença de SM } & \multirow{2}{*}{ Total } \\
\hline & & Não & Sim & \\
\hline \multirow[t]{2}{*}{ Privada } & 9 & $109(88,6 \%)$ & $14(11,4 \%)$ & $123(100 \%)$ \\
\hline & 8 & $69(83,1 \%)$ & $14(16,9 \%)$ & $83(100 \%)$ \\
\hline \multirow[t]{2}{*}{ Pública } & 9 & $60(80,0 \%)$ & $15(20,0 \%)$ & $75(100 \%)$ \\
\hline & $\delta$ & $37(66,1 \%)$ & $19(33,9 \%)$ & $56(100 \%)$ \\
\hline Total & & $275(81,6 \%)$ & $62(18,4 \%)$ & $337(100 \%)$ \\
\hline
\end{tabular}

Fonte: Autores 
Quanto à presença de histórico familiar, pôde-se observar na tabela 3 que nas escolas públicas apenas $14,7 \%$ dos adolescentes que não relataram histórico da presença de fatores de risco também apresentaram a SM, em contraste com $85,3 \%$ de portadores nos que tinham histórico familiar de presença de fatores de risco. Resultados similares ocorreram nas escolas privadas: $11,3 \%$ dos que não possuíam histórico eram portadores de SM e 92,8\% dos que tinham histórico familiar de risco apresentaram SM.

Tabela 3 - Porcentagem de alunos portadores de Síndrome Metabólica (SM) segundo a presença ou não de histórico familiar de fatores de risco.

\begin{tabular}{llll}
\hline \multirow{2}{*}{ Escola } & \multicolumn{2}{c}{ Presença de SM } & \multirow{2}{*}{ Total } \\
& Sem histórico & Com histórico & \\
\hline Pública & $5(14,7 \%)$ & $29(85,3 \%)$ & $34(100,0 \%)$ \\
Privada & $2(7,1 \%)$ & $26(92,8 \%)$ & $28(100,0 \%)$ \\
Total & $7(11,3 \%)$ & $55(88,7 \%)$ & $62(100,0 \%)$ \\
\hline
\end{tabular}

p-valor $=0,0483$

Fonte: Autores

Quando os parâmetros bioquímicos e IMC são analisados separadamente verifica-se que as porcentagens de estudantes que já apresentaram alguma alteração nos níveis considerados seguros (isto é, possuem glicemia aumentada, dislipidemia, obesidade ou sedentarismo) foram bastante alarmantes (Tabela 4): 16,9\% apresentaram glicemia aumentada; 27,0 apresentaram níveis aumentados de colesterol total; $15,5 \%$ mostraram níveis elevados de triacilglicerídeos; $15,4 \%$ e $18,1 \%$ apresentaram níveis alterados de LDL-c e HDL-c respectivamente; $15,4 \%$ eram obesos e $54,0 \%$ sedentários.

Tabela 4 - Porcentagens dos estudantes das escolas privadas e públicas que apresentaram valores aumentados na glicemia, nos níveis de colesterol total e frações e nos níveis de triglicerídeos e que apresentaram obesidade e sedentarismo.

\begin{tabular}{l|c|c}
\hline Parâmetros alterados & $\mathrm{n}^{\circ}$ estudantes & $\%$ \\
\hline Glicose $>99 \mathrm{mg} / \mathrm{dL}$ & 57 & 16,9 \\
$\mathrm{CT}>150 \mathrm{mg} / \mathrm{dL}$ & 91 & 27,0 \\
$\mathrm{TGC}>100 \mathrm{mg} / \mathrm{dL}$ & 59 & 15,5 \\
LDL-c $>100 \mathrm{mg} / \mathrm{dL}$ & 52 & 15,4 \\
HDL-c $<45 \mathrm{mg} / \mathrm{dL}$ & 61 & 18,1 \\
Obesidade & 52 & 15,4 \\
Sedentários & 182 & 54,0 \\
\hline
\end{tabular}

Fonte: Autores 


\section{Discussão}

A presença de SM ou fatores isolados de risco vascular acometendo jovens aparentemente hígidos, como foi observado neste trabalho, (Tabela 1, 2 e 4) corrobora outros estudos da literatura (AALTO et al., 2012; ATABEK, 2011). Cook et al. (2003) estimou que a prevalência global de SM em adolescentes é de aproximadamente $4 \%$, com um aumento de $29 \%$ em adolescentes com excesso de peso, mas os resultados deste trabalho evidenciaram proporções maiores. De acordo com Sarrafzadegan et al. (2013) a obesidade tornou-se um grave problema de saúde em crianças, que está associada com disfunção metabólica significativa, e que acompanha a vida adulta. A obesidade é um fator importante preditor da SM.

$\mathrm{O}$ fato de ser encontrado número expressivo de adolescentes com SM, tanto na rede pública quanto privada, pode ser justificado pelas mudanças no estilo de vida e hábitos alimentares tanto nas classes sociais menos favorecidas, quanto nas mais privilegiadas. Estudo mostra que nos últimos anos, houve, no Brasil, aumento no consumo de gorduras, carnes, leite e derivados ricos em gorduras, contribuindo com o aumento da densidade energética ingerida. As mudanças dietéticas ganharam maior repercussão no Brasil principalmente a partir da década de 80 , em que houve aumento no consumo de ácidos graxos saturados, açúcares e refrigerantes, em detrimento da redução da ingestão de carboidratos complexos, verduras, legumes e frutas, principalmente nas regiões metropolitanas do Brasil (MIRMIRAN, 2009).

A substituição dos alimentos naturais ricos em fibras, vitaminas e minerais pelo crescente consumo de produtos industrializados, associada ao sedentarismo, mudanças na estrutura de trabalho e avanços tecnológicos, compõem um dos os principais fatores etiológicos para deposição de gordura visceral, obesidade e complicações futuras como o aumento do risco de doenças (SILVA et al.,
2010).

Pode-se entender o elevado número de indivíduos com SM, ou mesmo já com fatores de riscos predisponentes para ela na população de baixa renda, devido ao elevado consumo de alimentos hipercalóricos como os farináceos, óleos, gorduras e açúcar simples relacionados a um gasto energético inferior ao consumido. Em contrapartida, nas classes com melhor poder aquisitivo a ingestão de alimentos mais elaborados de alta densidade energética, carboidratos refinados, gorduras saturadas, ácidos graxos trans, colesterol e alimentos tipo fast food, associados à redução da atividade física implica no ganho de peso da população, predispondo ao surgimento dos fatores de risco e consequentemente na crescente incidência da SM (MENDOZA; WATSON; CULLEN, 2010).

Os resultados deste trabalho também mostram que há relação entre a presença de fatores de risco de SM e histórico familiar (Tabela 3) independente da classe social. No entanto, a incidência de estudantes com história familiar positiva para a SM neste estudo foi estatisticamente maior nas escolas públicas. Este fato pode estar associado ao consumo de alimentos mais elaborados e mais ricamente energéticos. Outros autores tiveram resultados semelhantes como pode ser visto por Ricardo, Caldeira e Corso. (2009) que encontraram níveis maiores de obesos em escolas particulares no sul do Brasil. A interação genética/nutrição também pode ser determinante de um largo espectro de consequências antropométricas e metabólicas e, nestes casos, a prevenção dos danos vasculares pode ser feita com um programa de educação alimentar com aumento do consumo de frutas e verduras e perda de peso. As mudanças de hábito alimentar podem se relacionar diretamente à perda de peso nas crianças e demais faixas etárias e redução dos fatores de risco vascular (NAKAYAMA, 2010; MENDOZA; WATSON; CULLEN, 2010).

A prática de atividade física também é importante na prevenção dos fatores de risco de SM 
assim como pode diminuir os índices de obesidade (MARTÍNEZ-GÓMEZ et al., 2009). A tabela 4 mostra que mais da metade dos estudantes avaliados tem vida sedentária e que mais de $15 \%$ deles podem ser considerados obesos (ENES et al., 2009). Estes níveis de obesidade tendem a crescer em razão da elevada prevalência de sedentarismo. Tenório (2010) também investigou o comportamento de adolescentes em relação à atividade física e relatam que há altos níveis de sedentarismo (ainda maiores dos que os observados neste trabalho) entre os jovens e que muitos gastam boa parte de seu tempo com televisão, videogame e computador ao invés de participarem de atividades desportivas.

O sexo também pode influenciar no desenvolvimento das dislipidemias e fatores de riscos cardiovasculares. Há inúmeras evidências de que as mulheres têm menores chances de risco do que os homens indicando que os hormônios sexuais modulam muitos fatores endócrinos envolvidos na aterosclerose (PÉREZ-LOPEZ et al., 2010). A observação da tabela 1 também indica que já existem diferenças significativas dos fatores de risco quando se comparam os sexos. Apesar dos indivíduos do sexo feminino terem apresentado maiores médias de pressão arterial e glicemia e menores médios para os valores de HDL-c, quando se fez o cômputo geral, observou-se que houve maior prevalência de SM no sexo masculino (tabela 2).

Diante da atual epidemia mundial de SM que acomete cada vez mais faixas etárias mais novas das populações, a realidade da cidade estudada não é diferente de outras cidades brasileiras (RINALDI et al., 2010) ou de outros países (BAYA-BOTTI et al., 2009). É importante que as autoridades entendam o que é esta epidemia e quais são as suas repercussões na sociedade e para o sistema de saúde.

Os resultados deste trabalho mostram que há elevada incidência de SM principalmente nos estudantes das escolas públicas avaliadas. As porcentagens alcançam os $20,0 \%$ no sexo feminino e ultrapassam os $30 \%$ nos indivíduos do sexo masculino. Sendo assim, é de extrema importância a orientação dos escolares e seus familiares quanto aos maus hábitos alimentares e sedentarismo, assim como suas consequências. Outras medidas podem ser tomadas na própria escola, que é o local onde o jovem passa um tempo substancial do seu dia. A escolha de um cardápio mais saudável pode colaborar de forma significativa no comportamento alimentar do adolescente dentro do ambiente escolar, o que pode ser extrapolado também para sua conduta em casa. Devido às crescentes mudanças na Medicina, de intervencionista à preventiva, tais medidas tornam-se plausíveis e indispensáveis.

\section{Conclusão}

A SM tem alta prevalência na população estudada, o que é preocupante já que o grupo estudado constitui-se de adolescentes. Sendo assim, são necessárias medidas intervencionistas urgentes, abordadas por equipe multidisciplinar para que os adolescentes sejam assistidos integralmente para que se tornem indivíduos adultos mais saudáveis.

\section{Referências}

AALTO, K.; MAKSIMOW, M.; JUONALA, M.; VIIKARI, J.; JULA, A.; KÄHÖNEN, M.; JALKANEN, S.; RAITAKARI, O. T.; SALMI, M. Soluble vascular adhesion protein-1 correlates with cardiovascular risk factors and early atherosclerotic manifestations. Arteriosclerosis, Thrombosis, and Vascular Biology, Dallas, v. 32, n. 2, p.523-32, 2012.

ATABEK, M. E. Non-alcoholic fatty liver disease and metabolic syndrome in obese children. World Journal of Gastroenterology, Beijing v. 17, n. 39, p. 4445-6, 2011.

BAYA-BOTTI, A.; PÉREZ-CUETOM, F. J.; VASQUEZ MONLLORM, P.A.; KOLSTEREN, P. W. Anthropometry of height, weight, arm, wrist, abdominal circumference and body mass index, for Bolivian adolescents 12 to 18 years: Bolivian adolescent percentile values from the MESA study. Nutrición Hospitalaria, Madrid, v. 24, n. 3, p. 304-311, 2009. 
BORNEO, R.; LEÓN, A. E. Whole grain cereals: functional components and health benefits. Food \& Function, Cambridge, v. 3, n. 2, p. 110-119, 2012.

COOK, S; WEITZMAN, M.; AUINGER, P.; NGUYEN, M.; DIETZ, W. H. Prevalence of a metabolic syndrome phenotype in adolescents: findings from the third National Health and Nutrition Examination Survey, 1988-1994. Archives of Pediatrics \& Adolescent Medicine, Chicago, v. 157, p. 821-7, 2003.

DEMARCO, M.; DE SIMONE, G.; IZZO, R.; MANCUSI, C.; SFORZA, A.; GIUDICE, R.; TRIMARCO, B.; DE LUCA, N. Classes of antihypertensive medications and blood pressure control in relation to metabolic risk factors. Journal of Hypertension, London, v. 30, n. 1, p. 188-93, 2012.

EL MESALLAMY, H. O.; EL-DEMERDASH, E.; HAMMAD, L. N.; EL-MAGDOUB, H. M. Effect of taurine supplementation on hyperhomocysteinemia and markers of oxidative stress in high fructose diet induced insulin resistance. Diabetology and Metabolic Syndrome, London, v. 30, n. 1, p. 46, 2010.

ENES, C. C.; FERNANDEZ, P. M. F.; VOCI, S. M.; TORAL, N.; ROMERO, A.; SLATER, B. Validade e confiabilidade das medidas referidas de peso e estatura para o diagnóstico do estado nutricional de adolescentes. Revista Brasileira de Epidemiologia, São Paulo, v. 12, n. 4, p. 627-635, 2009.

HEYWARD, H. V.; STOLAZARCZYK, M. L. Métodos de dobras cutâneas In: . Avaliação da composição corporal aplicada. São Paulo: Manole, 2000.

MARTÍNEZ-GÓMEZ, D.; EISENMANN, J. C.; MOYA, J. M.; GÓMEZ-MARTÍNEZ, S.; MARCOS, A.; VEIGA, $\mathrm{O}$. L. The role of physical activity and fitness on the metabolic syndrome in adolescents: effect of different scores, The AFINOS Study. Journal of Physiology and Biochemistry, Panplona, v. 65, n. 3, p. 277-89, 2009.

MENDOZA, J. A.; WATSON, K.; CULLEN, K. W. Change in dietary energy density after implementation of the texas public school nutrition policy. Journal of the Academy of Nutrition and Dietetics, New York, v. 110, n. 3, p. 434-40, 2010.

MIRMIRAN, P. Fruit and vegetable consumption and risk factors for cardiovascular disease. Metabolism, New York, v. 58, n. 4, p. 460-468, 2009.

NAKAYAMA, T. Genetic polymorphisms of prostacyclin synthase gene and cardiovascular disease. The International Journal of Angiology, Nesconset, v. 29, n. 2, p. 33-42, 2010.
OH, J.; KIM, J. Y.; PARK, S.; YOUN, J. C.; SON, N. H.; SHIN,D. J.; LEE, S. H.; JEE, S. H.; JANG, Y. The relationship between insulin-like growth factor-1 and metabolic syndrome, independent of adiponectin. Clinica Chimica Acta, Amsterdam, v. 13, n. 3-4, p. 50610, 2012.

PÉREZ-LÓPEZ, F. R.; LARRAD-MUR, L.; KALLEN, A.; CHEDRAUI, P.; TAYLOR, H. S. Gender differences in cardiovascular disease: hormonal and biochemical influences. Reproductive sciences, Thousand Oaks, v. 17, n. 6, p. 511-31, 2010.

RICARDO, G. D.; CALDEIRA, G. V.; CORSO, A. T. Prevalência de sobrepeso e obesidade e indicadores de adiposidade central em escolares de Santa Catarina, Brasil. Revista Brasileira de Epidemiologia, São Paulo, v. 12, n. 3, p. 424-435, 2009.

RINALDI, A. E.; PIMENTEL, G. D.; PEREIRA, A. F.; GABRIEL, G. F.; MORETO, F.; BURINI, R. C. Metabolic syndrome in overweight children from the city of Botucatu - São Paulo State - Brazil: agreement among six diagnostic criteria. Diabetology \& metabolic syndrome, London, v. 2, p. 1, p: 39-47, 2010.

SÁNCHEZ-GÓMEZ, J. M.; MARTÍNEZ-DOLZ, L.; SÁNCHEZ-LÁZARO, I.; ALMENAR, L.; SÁNCHEZLACUESTA, E.; MUÑOZ-GINER, B.; PORTOLÉS, M.; RIVERA, M.; VALERA-ROMÁN, A.; GONZÁLEZJUANATEY, J. R.; TEJADA-PONCE, D.; AGÜERO, J.; BUENDÍA, F.; SALVADOR, A. Influence of metabolic syndrome on development of cardiac allograft vasculopathy in the transplanted heart. Transplantation, Baltimore, v. 93, n. 1, p. 106-11, 2012.

SILVA, J. V. L.; TIMÓTEO, A. K. C. D.; SANTOS, C. D.; FONTES, G.; ROCHA, E. M. M. Consumo alimentar de crianças e adolescentes residentes em uma área de invasão em Maceió, Alagoas, Brasil. Revista Brasileira de Epidemiologia, São Paulo, v. 13, n. 1, p. 83-93, 2010.

SARRAFZADEGAN,N.;GHARIPOUR,M.; SADEGHI, M.; NOURI, F.; ASGARY, S.; ZARFESHANI, S. Differences in the prevalence of metabolic syndrome in boys and girls based on various definitions. ARYA Atheroscler, Isfahan, ,v. 9, n. 1, p. 70-6, 2013.

SOCIEDADE BRASILEIRA DE CARDIOLOGIA. V Diretrizes brasileiras de hipertensão arterial. Arquivos Brasileiros de Cardiologia, São Paulo, v. 89, n. 3, p. 2479, 2007.

SOCIEDADE BRASILEIRA DE CARDIOLOGIA. I Diretriz de prevenção da arterosclerose na infância. Arquivos Brasileiros de Cardiologia, São Paulo, v. 85, n.6, p. 3-36, 2005. 
STEVENS, G. A.; KING, G.; SHIBUYA, K. Deaths from heart failure: using coarsened exact matching to correct cause-of-death statistics. Population Health Metrics, London, v. 13, p.8-16, 2010.

TENÓRIO, M. C. M. Atividade física e comportamento sedentário em adolescentes estudantes do ensino médio. Revista Brasileira de Epidemiologia, São Paulo, v.13, n. 1, p. 105-117, 2010. 
